\title{
Interpretación textural de dos clínkeres de cemento portland de distinta velocidad de fraguado
}

\author{
ANGEL RODRIGUEZ REY \\ Departamento de Petrología \\ Universidad de Oviedo
}

\section{$R E S U M E N$}

En el presente trabajo se ha realizado un estudio textural, mediante microscopia óptica de reflexión, de dos clinkeres industriales de cemento portland procedentes del mismo horno, que se diferenciaban por poseer velocidades de fraguado muy distintas. Se observó la existencia de marcadas diferencias mineralógicas-texturales entre ellos, debidas a sus distintas condiciones de enfriamiento, como se ha podido deducir de la interpretación genética de sus texturas.

\author{
$S U M M A R Y$ \\ Two industrial portland cement clinkers from the same \\ kiln, with different setting rates, were texturally \\ characterizated by reflected light microscopy. \\ They showed remarkable mineralogic and textural \\ differences which arised during their distinct \\ cooling processes, according to the interpretation \\ of their textures.
}

\section{INTRODUCCION}

Se ha realizado un estudio comparativo de la textura de dos clínkeres industriales de cemento portland procedentes del mismo horno - de vía seca- que presentan una marcada diferencia en su velocidad de fraguado, uno de fraguado normal, F.N., y otro de fraguado lento, F.L.

En la tabla I se recogen las caracteristicas del fraguado de ambos clínkeres, así como las resistencias mecánicas de sus morteros normalizados, siendo estas últimas muy similares a pesar de las mencionadas diferencias en la velocidad de fraguado.

\section{PREPARACION DE MUESTRAS}

De ambos clínkeres se prepararon secciones pulidas aptas para su observación mediante microscopía de reflexión. Para ello se ha seguido, con escasas modificaciones, el procedimiento descrito en (1). Se puso especial cuidado en coger un número representativo de gránulos de cada una de las fracciones granulométricas establecidas previamente por el tamizado. Los gránulos, convenientemente desbastados y pulidos, se observarón por luz reflejada, sin atacar primero, y trás el ataque con etanol-agua $(1: 1)$ seguido del de una disolución al $1 \%$ de $\mathrm{ClH}$ en etanol. 
TAB LA I

Composición química de los dos clínkeres. Propiedades fisicas de sus cementos, y propiedades mecánicas de los morteros normalizados

\begin{tabular}{|c|c|c|c|}
\hline & & CLINKER F.N. & CLINKER F.L. \\
\hline $\begin{array}{l}\text { Tiempo de fraguado (min) } \\
\text { (aguja de vicat) }\end{array}$ & $\left\{\begin{array}{l}\text { inicial } \ldots \ldots \ldots \ldots \ldots \ldots \\
\text { final } \ldots \ldots \ldots \ldots \ldots \ldots\end{array}\right.$ & $\begin{array}{l}135 \\
210\end{array}$ & $\begin{array}{l}270 \\
350\end{array}$ \\
\hline $\begin{array}{l}\text { Finura de molido. Residuo } \\
\text { sobre tamiz (mallas } / \mathrm{cm}^{2} \text { ) }\end{array}$ & $\left\{\begin{array}{r}900 \ldots \ldots \ldots \ldots \ldots \ldots \\
4.900 \ldots \ldots \ldots \ldots \ldots\end{array}\right.$ & $\begin{array}{l}0,2 \% \\
1,6 \%\end{array}$ & $\begin{array}{l}0,0 \% \\
0,2 \%\end{array}$ \\
\hline \multirow{2}{*}{\multicolumn{2}{|c|}{$\begin{array}{l}\text { Superficie espec. }\left(\mathrm{cm}^{2} / \mathrm{g}\right) \ldots \ldots \ldots \ldots \ldots \ldots \ldots \ldots \ldots \ldots \ldots \\
\text { Agua pasta cons. normal } \ldots \ldots \ldots \ldots \ldots \ldots \ldots \ldots \ldots \ldots\end{array}$}} & 3.041 & 3.320 \\
\hline & & $24,0 \%$ & $25,4 \%$ \\
\hline Resistencias mecánicas $\left(\mathrm{kp} / \mathrm{cm}^{2}\right)$ & $1 \mathrm{~d} \ldots \ldots \ldots \ldots \ldots$ & $169 / 47$ & $171 / 43$ \\
\hline \multirow{3}{*}{\multicolumn{2}{|c|}{$\begin{array}{l}3 \mathrm{~d} \ldots \ldots \ldots \ldots \ldots \ldots \ldots \\
7 \mathrm{~d} . \ldots \ldots \ldots \ldots \ldots \ldots \\
28 \mathrm{~d} . \ldots \ldots \ldots \ldots \ldots \ldots\end{array}$}} & $373 / 61$ & $314 / 67$ \\
\hline & & $384 / 78$ & $366 / 75$ \\
\hline & & $499 / 86$ & $511 / 80$ \\
\hline \multicolumn{4}{|l|}{ Composición quimica clínker (\%) } \\
\hline \multicolumn{2}{|l|}{ Pérdida al fuego ... } & 0,5 & 0,3 \\
\hline \multicolumn{2}{|l|}{ Residuo insoluble . } & 0,1 & 0,1 \\
\hline \multicolumn{2}{|l|}{ Oxido de Magnesio ........... } & 2,9 & 3,2 \\
\hline \multicolumn{2}{|c|}{ 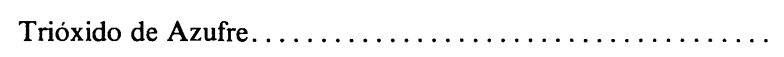 } & 1,0 & 0,7 \\
\hline \multicolumn{2}{|l|}{ Dióxido de Silicio....... } & 20,3 & 20,9 \\
\hline \multicolumn{2}{|c|}{ Oxido de Aluminio $\ldots \ldots \ldots \ldots \ldots \ldots \ldots \ldots \ldots \ldots$} & 6,5 & 6,6 \\
\hline \multicolumn{2}{|c|}{ Oxido de Hierro $\ldots \ldots \ldots \ldots \ldots \ldots \ldots \ldots \ldots \ldots \ldots \ldots \ldots$} & 3,5 & 3,2 \\
\hline \multicolumn{2}{|c|}{ Oxido de Calcio.$\ldots \ldots \ldots \ldots \ldots \ldots \ldots \ldots \ldots \ldots \ldots \ldots$} & 64,6 & 64,4 \\
\hline \multicolumn{2}{|c|}{ 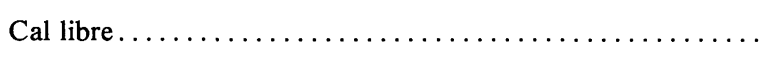 } & 1,0 & 0,7 \\
\hline
\end{tabular}

\section{ANALISIS CUANTITATIVO}

Se llevó a cabo utilizando un contador de puntos SWIFT, con saltos de $167 \mu \mathrm{m}$ en recorridos horizontales distanciados en $400 \mu \mathrm{m}$, de forma que se superponía una red ortogonal a toda la sección de cada gránulo contado. Los datos obtenidos se expresan en la tabla II.

\section{DESCRIPCIÓN TEXTURAL}

\subsection{Clinker F.N.}

Coincidiendo con los datos del análisis modal, se observa una proporción relativamente baja de belita, siendo la porosidad también baja y la cal libre primaria escasa.

La distribución de las fases es bastante homogénea. La belita está uniformemente repartida en general (foto 1), aunque en algunos gránulos se observan pequeños nidos de este mineral; estos mismos gránulos poseen también pequeños nidos de cal libre (foto 2 ). 


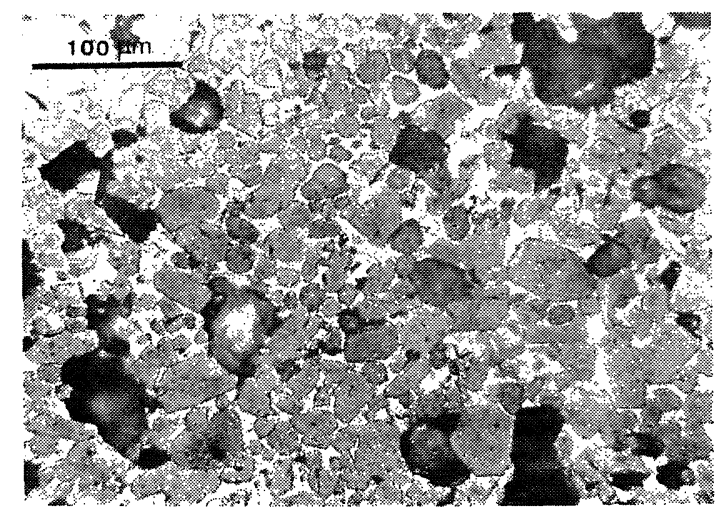

Foto 1.-Aspecto general del clinker F.N. La belita, en granos de pequeño tamaño, está homogéneamente repartida. Obsérvese el carácter heterogranular de la alita. (× 210).

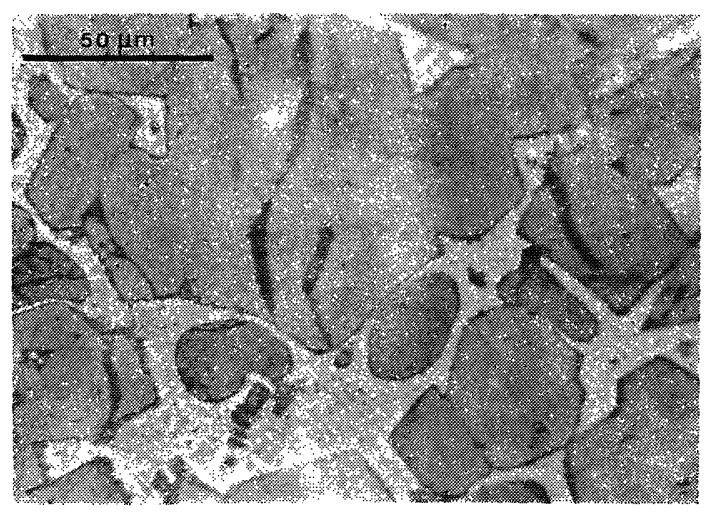

Foto 3.-Alitas en sineusis. En los ángulos superior derecho e inferior izquierdo, belita secundaria orlando a las alitas, que aparece también en la matriz. Los cristales de ferrito y de aluminato están poco desarrollados. Clínker F.N. ( $\times 530)$.

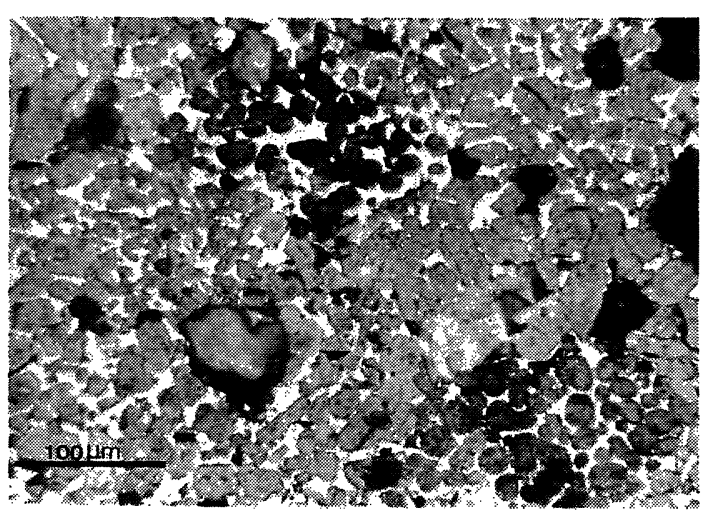

Foto 2.-Clinker de fraguado normal: dos pequeños nidos, uno de belita y otro de cal libre primaria, muy próximos entre sí. ( $\times 210)$.

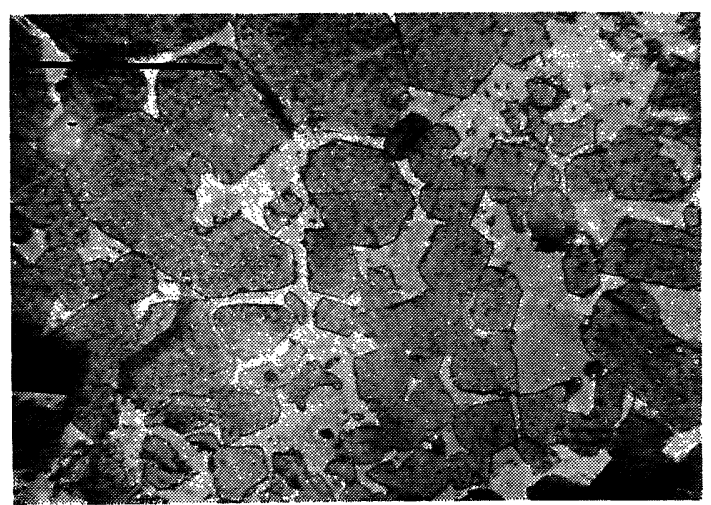

Foto 4.-Grandes placas de aluminato en la matriz del clinker de fraguado normal. También se observan pequeñas orlas de belita secundaria alrededor de algunos cristales de alita. (x 530).

La alita se presenta en cristales idiomórficos y subidiomórficos heterogranulares. Es frecuente que varios cristales crezcan juntos en sineusis (foto 3 ).

La belita aparece en cristales de forma redondeada con maclas polisintéticas cruzadas. En algunos gránulos se observa, no obstante, una incipiente transformación a belita lobulada. El tamaño de los cristales en sección pulida oscila entre las 10 y las $20 \mu \mathrm{m}$, situándose la media alrededor de las $15 \mu \mathrm{m}$. Aunque no es muy frecuente, en ciertos gránulos se observa, tanto incluida en la matriz como orlando a cristales de alita, cierta cantidad de belita secundaria que adopta en ocasiones formas dendríticas (foto 3 ).

La proporción de matriz es apenas inferior a la normal. Las fases que la constituyen presentan un desarrollo aceptable, aunque muy variable; en algunos gránulos, el aluminato forma grandes placas (foto 4).

\subsection{Clínker F.L.}

A diferencia del anterior, este clinker presenta una distribución heterogénea de las fases que lo constituyen. Son muy abundantes los nidos y aglomeraciones de belita, a veces de forma 
irregular y de gran tamaño (hasta $1,5 \mathrm{~mm}$ de largo). También aparecen nidos de cal libre primaria, no siempre muy compactos, situados generalmente próximos a los de belita (foto 5). Por otra parte, tanto el contenido de ferrito y aluminato como la porosidad, son bajos.

La alita se presenta en cristales idiomórficos y subidiomórficos de tamaño muy variado, algunos de ellos muy corroidos (foto 6). Los más grandes aparecen generalmente intercrecidos en sineusis (foto 5). Los cristales subidiomórficos presentan rebordes de belita secundaria a veces de gran tamaño.

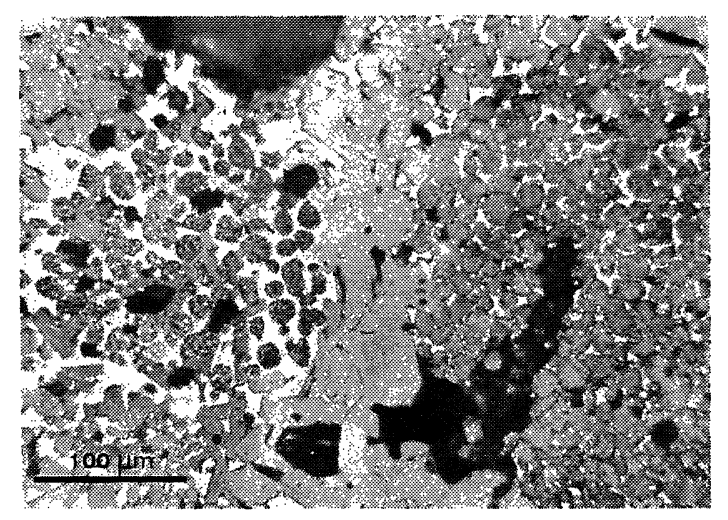

Foto 5.-Nido compacto de belita separado por una franja de grandes cristales de alita, de una concentración poco compacta de cal libre. Clinker F.L. ( 210$)$.

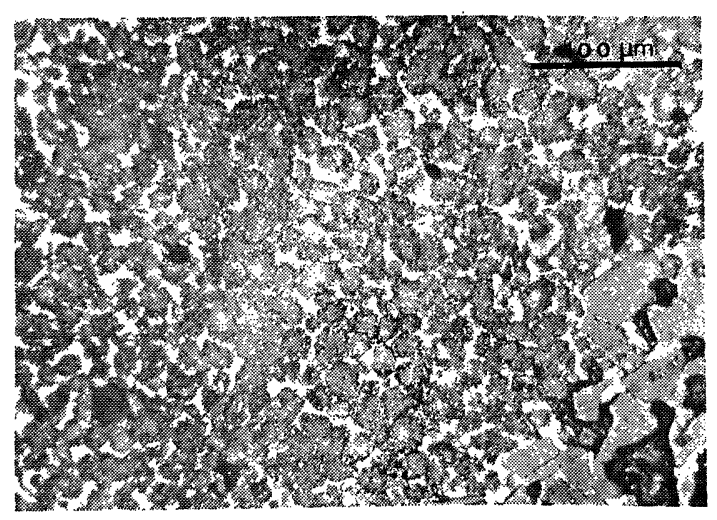

Foto 7.-Nido de belitas lobuladas en el clinker de fraguado lento. $(\times 210)$.

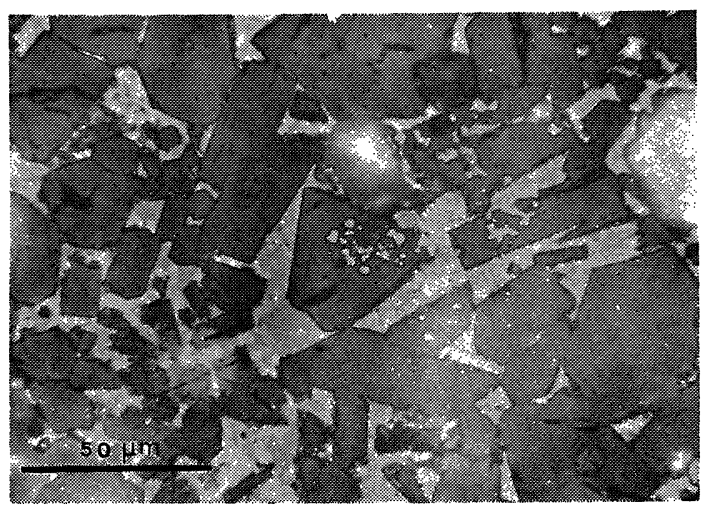

Foto 6.-Clinker F.L. Cristales de alita subidiomórficos corroidos. Obsérvese el gran desarrollo del ferrito y del aluminato. $(\times 530)$.

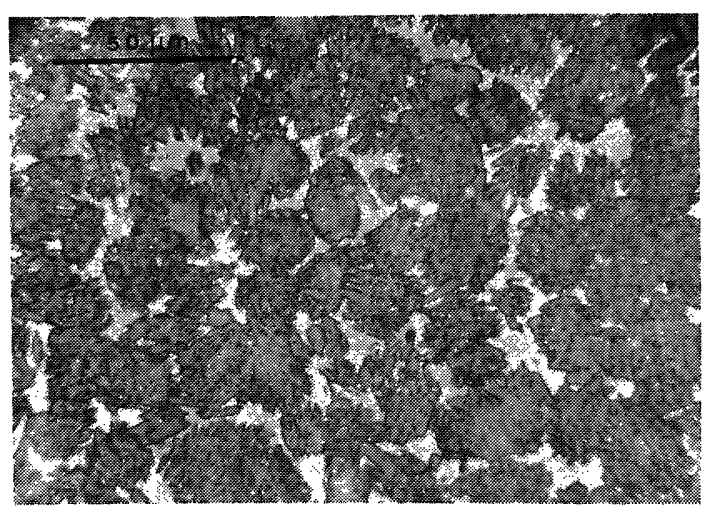

Foto 7a.-En detalle se observa la importante modificación que ha sufrido la belita primaria del clinker de fraguado lento, adoptando como consecuencia formas esqueléticas. $(\times 530)$

La belita, más abundante que en el clínker de fraguado normal, aparece con formas anómalas. Por un lado, la de carácter primario se presenta en su mayor parte con formas y contornos lobulados (fotos 7 y $7 \mathrm{a}$ ); son visibles todavia dos sistemas de maclas que se disponen entrecruzadas. Tal como se ha mencionado anteriormente, este mineral se dispone en aglomeraciones y nidos. Los cristales son más o menos de tamaño similar, situándose la media alrededor de las $15 \mu \mathrm{m}$. Por otra parte, hay gran cantidad de belita secundaria dendritica procedente de la descomposición de la alita (fotos 8 y 8a); este tipo de belita exhibe formas dendríticas muy bien desarrolladas que se presentan, bien orlando a los cristales de alita a los que corroen, bien rodeados completamente por matriz. 


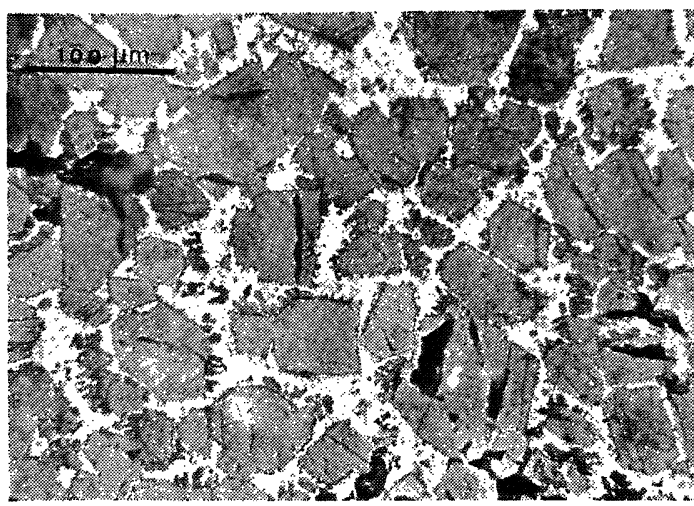

Foto 8.-Aspecto parcial de uno de los gránulos del clinker F.L., donde se observa gran cantidad de belita secundaria que corroe a los cristales de alita. ( $\times 210)$.

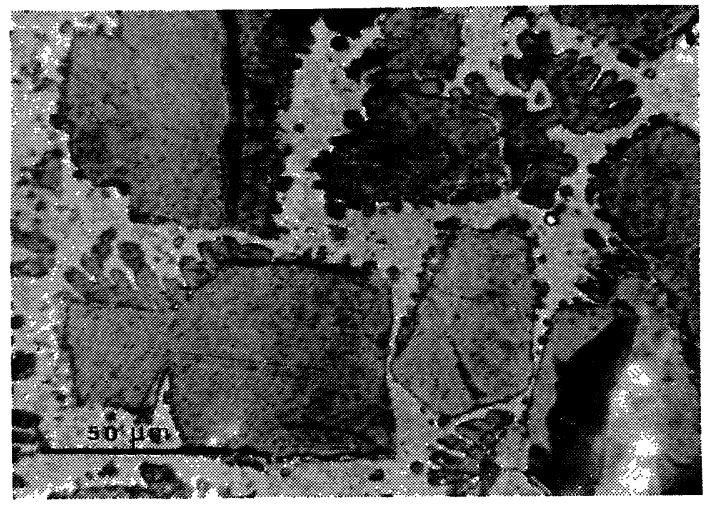

Foto $8 \mathrm{a}$.-Idem, detalle de las citadas belitas secundarias con formas dendriticas perfectamente desarrolladas. (x 530).

El desarrollo de los constituyentes de la matriz es variable, observándose en muchos casos grandes placas tanto de ferrito como de aluminato (foto 6).

\section{INTERPRETACION TEXTURAL}

Las condiciones de los procesos de preparación y de cocción del crudo del clínker de fraguado normal han sido apropiadas, aunque dado el tamaño, un poco pequeño de la belita; quizás el tiempo de permanencia en el horno haya sido algo escaso (2). El tamaño medio de la alita, de unas 30-35 $\mu \mathrm{m}$, indica una velocidad de calentamiento correcta (2).

La molturación y la homogeneización del crudo son buenas, puesto que a pesar de ciertos nidos de belita y de cal libre primaria en algunos gránulos, la distribución de las fases es, por lo general, bastante homogénea.

El clinker de fraguado lento ha seguido un proceso de cocción similar. Sin embargo, la presencia de grandes y abundantes aglomeraciones y nidos de belita, además de algunos de cal libre, denota irregularidades en la molturación y la homogeneización del crudo.

Las diferencias entre ambos clínkeres se originan, principalmente, durante el enfriamiento. E1 de fraguado normal experimentó un enfriamiento correcto, aun cuando en algunos gránulos se observan indicios de belitas lobuladas o de belitas secundarias dendriticas. El de fraguado lento sufrió un enfriamiento anómalo que se refleja, sobre todo, en las caracteristicas de la belita: la de carácter primario se presenta con formas típicamente lobuladas, resultado de la transformación de la belita primaria habitual en los clinkeres industriales, redondeada y con maclas polisintéticas cruzadas; esta transformación requiere tiempo y sólo es posible en clínkeres enfriados lentamente, tal como se menciona en (3). Hay, además, gran cantidad de belita secundaria dendritica muy bien desarrollada que procede de la descomposición de la alita en un proceso lento por debajo de los $1.250^{\circ} \mathrm{C}$, como se señala en (1).

\section{CONCLUSIONES}

- La observación microscópica ha puesto de manifiesto las diferencias mineralógico-texturales entre los dos clínkeres estudiados. 
- De la interpretación genética de la textura, se deduce que estas diferencias se deben a las distintas características del enfriamiento experimentado por cada clínker, correcto el del F.N. y anormalmente lento el sufrido por el F.L.

- Dada la gran similitud en la composición química de ambos clínkeres y en las características de molido y amasado de sus pastas, es muy probable la influencia de estas diferencias mineralógico-texturales en el hecho de que los dos clínkeres presenten tan marcadas diferencias en el fraguado. Por otra parte, también puede contribuir a ello la diferencia en la composición mineralógica (más belita y menos alita en el clínker F.L.).

\section{BIBLIOGRAFIA}

(1) FERNANDEZ PARIS, J. M. (1968): "microscopia del clinker de cemento portland". Manuales y Normas del Instituto Eduardo Torroja de la Construcción y del Cemento. Madrid.

(2) ONO, Y. (1981): "Microscopical observation of clinker for the estimation of burning condition, grindability and hydraulic activity". Proceed. of the 3rd Int. Conf. on Cement Microscopy. Edited by G. R. Gouda. Texas. pp. 198-210.

(3) GILLE, F., DREIZLER, I., GRADE, K., KRAMER, H. y WOERMANN, E. (1965): “Mikroskopie des Zementklinkers. Bilderatlas”. Editado por la Agrupación Alemana de Fábricas de cemento. Beton- Verlag. Düsseldorf. (Suplemento, versión española de J. Calleja. IETcc., Madrid).

\section{RECONOCIMIENTO}

El autor desea expresar su sincero agradecimiento a D. Enrique Fernández Molina de la Scdad. Tudela-Veguín (Asturias), por la valiosa aportación de los datos que se recogen en la Tabla I.

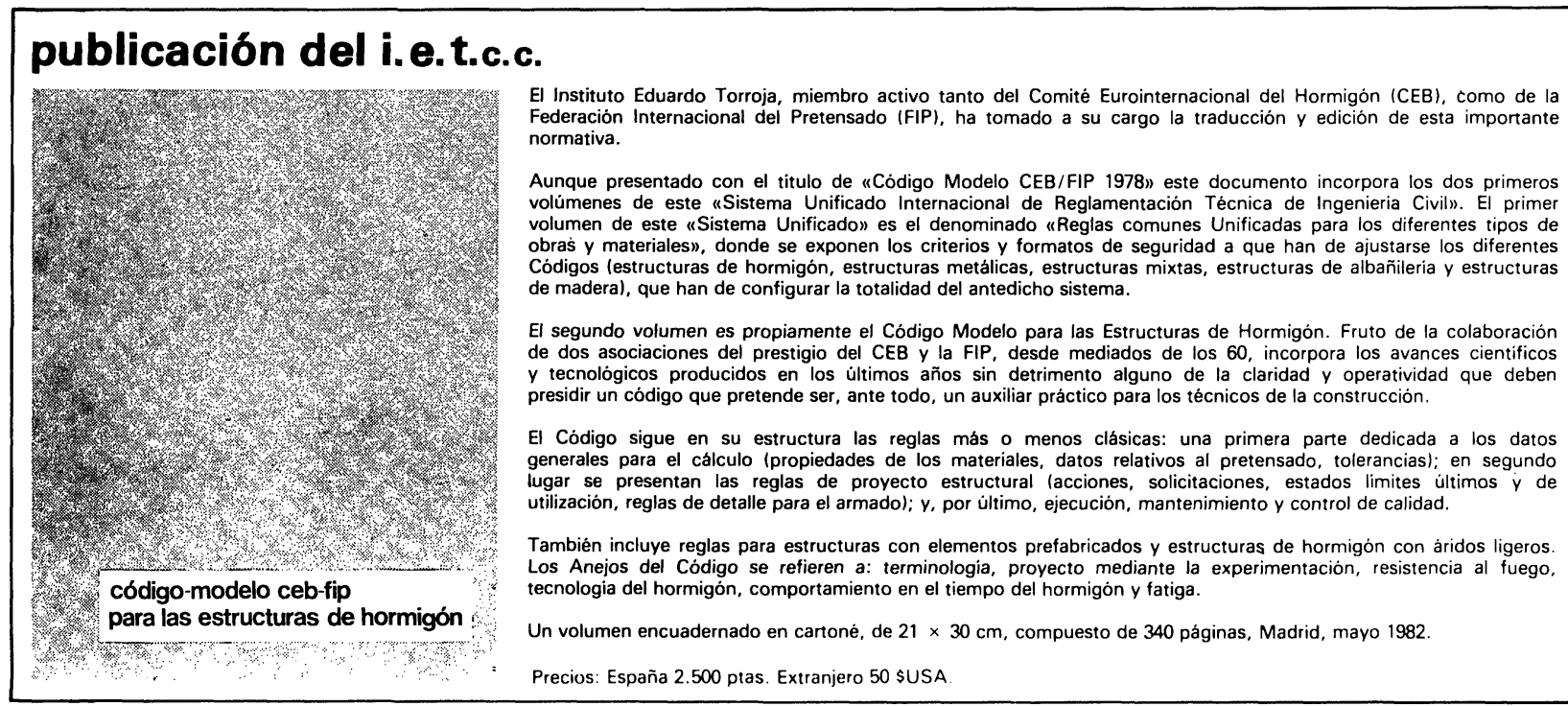

